\title{
2D Self-Assembled Molecular Networks and On-Surface Reactivity under Nanoscale Lateral Confinement
}

Received 00th January 20xx, Accepted 00th January 20xx DOI: $10.1039 / \times 0 \times x 00000 x$

\begin{abstract}
Lander Verstraete, ${ }^{* a}$ and Steven De Feyter*a
Supramolecular self-assembly at surfaces provides a pathway for building chemically customized interfaces. Over the last three decades, research on the role of key parameters such as temperature, solute concentration, and molecular design has enabled a steady increase in the complexity of self-assembled molecular networks (SAMNs) that can thus be created. However, the structure and quality of SAMNs is often determined during the early stages of nucleation and growth. To study and influence self-assembly processes at this deterministic length scale, spatial confinement of molecular adsorbates to well-defined surface patterns with nanoscale lateral dimensions offers exciting possibilities. The aim of this tutorial review is to give an overview of the various ways in which confinement impacts SAMN formation, and how we can use that knowledge to direct assemblies towards desired structures. The possibility to exploit confinement for improved control over on-surface reactions is also contemplated.
\end{abstract}

\section{Key Learning points}

- Surface patterns with nanoscale lateral dimensions limit the growth of 2D self-assembled molecular networks (SAMNs), such that confinement effects can be explored.

- SAMN formation in nanoscopic environments is characterized by altered assembly stabilities and diffusion dynamics, as well as possibly significant interactions with the confining host.

- The ability to isolate SAMNs at dimensions close to the critical nucleus size allows fundamental investigations on nucleation and growth mechanisms.

- Confinement can be used to obtain SAMNs with preferred molecular orientations, polymorphs or 2D enantiomorphs.

- Confinement of reactive precursor molecules enables improved product selectivity and size-control.

\section{Introduction}

The physical and chemical properties of materials confined within spatially restricted environments are often different from their bulk counterparts. These confinement effects emerge when material dimensions approach those of its constituting elements, but may persist at length scales far beyond. Within the field of supramolecular chemistry, we may

\footnotetext{
a. Department of Chemistry, Division of Molecular Imaging and Photonics, $K U$ Leuven-University of Leuven, Celestijnenlaan 200F, B-3001 Leuven, Belgium. Email: lander.verstraete@gmail.com, steven.defeyter@kuleuven.be
}

expect drastic changes to the kinetics and thermodynamics of spatially confined self-assembly processes as a result of impeded diffusion dynamics, size/geometry constraints, and specific interactions with the host medium. Knowledge of these confinement effects may allow to design specific templates for superior control over the fabrication of materials at the nanoscale.

While supramolecular self-assembly occurs in a variety of environments, the majority of studies on confinement effects have focused on crystallization from solution. Spatially confined crystals are commonly obtained by restricting crystal growth within a porous host such as porous glass, polymer matrices, or anodized aluminium oxide. Confinement in pores enforces large surface-to-volume ratios, which is reflected in the thermodynamic stability of crystals, especially at or near the critical nucleus size. An important consequence is sizedependent polymorphism, in which metastable phases become favored under nanoscale confinement. ${ }^{1}$ Furthermore, nanopores can be used to impact the nucleation behavior ${ }^{2}$ and crystal orientation. ${ }^{3}$ For a detailed account of the many ways in which confinement may impact crystallization from solution, the reader is referred to some outstanding review articles in the literature. ${ }^{4-6}$

Next to the solution phase, confinement effects can impact the formation of supramolecular assemblies formed at solid interfaces. Limited to two dimensions, molecules adsorbed on a crystalline surface can arrange themselves in a self-assembled molecular network (SAMN), whose structure is dictated by intermolecular and interfacial interactions. When interfacial interactions are non-covalent in nature, i.e. physisorption, the molecules generally lie flat on the surface. Naturally, the lack of a third dimension of such SAMNs represents a form of confinement, but this is not the focus of this review article. Rather, we consider confinement effects arising from self- 
assembly on specific surface templates or patterns with nanoscale lateral dimensions. Such patterns can occur naturally as a result of surface topography features such as step edges, but are mostly artificially created. For instance, selective oxidation, ${ }^{7}$ etching, ${ }^{8}$ or chemical modification ${ }^{9}$ are common strategies to generate effective surface patterns. In addition, a virtually unlimited number of pattern shapes can be fabricated via scanning probe lithography.

In this tutorial review, we introduce the advances made over the last three decades with regards to understanding the unique influences of lateral confinement on the formation of surface-supported SAMNs. We start this review with a brief description of classical 2D nucleation, as confined assemblies are often significantly impacted at this early stage. The main section then highlights different confinement effects induced by a variety of surface patterns, both in ultrahigh vacuum and ambient conditions. Next, we also focus on the potential of confinement to steer on-surface reactions. In all of these examples, scanning probe microscopies play a crucial role to characterize both the surface patterns and confined assemblies. The final section summarizes key aspects of the given examples, and contemplates future directions in this area.

\section{2D nucleation \& growth}

The initial stage of SAMN formation involves the formation of a nucleus, which can be defined as the smallest molecular cluster capable of independent existence. According to classical nucleation theory (CNT), which is well-established in the context of crystallization from solution, but which can also effectively be applied in 2D, these nuclei exhibit the same structure and density as the mature phase, and grow by sequential addition of monomeric units. This allows for a thermodynamic description of nucleation in terms of free energies. Assuming a circular nucleus with radius $r$, the free energy change associated with nucleation can be described by:

$$
\Delta G=\Delta G_{\text {interior }}+\Delta G_{\text {periphery }}=\pi r^{2} \cdot \Delta g+2 \pi r \cdot \sigma .
$$

In this expression, $\Delta g$ is the free energy difference per unit of area between the ordered and disordered phase, and $\sigma$ is the line tension of the interface between the nucleus and its surroundings. On the one hand, cluster formation is energetically favorable because of the intermolecular interactions that stabilize the assembly $(\Delta g<0)$. On the other hand, molecules at the periphery of the cluster are unstable with respect to those in the interior due to fewer binding partners $(\sigma>0)$. The net result is that nuclei exhibit a critical size, $r^{*}$, as shown in Fig. 1 . Below this critical size, the unfavorable line tension, $\sigma$, dominates and nuclei are bound to dissolve again, whereas above $r^{*}$, nuclei are stable and will grow spontaneously. Given the energy barrier, $\Delta G^{*}$, that must be overcome, the nucleation rate can be written as:

$$
J=A \exp \left(-\frac{\Delta G^{*}}{k T}\right),
$$

with $A$ a kinetic factor that depends on the molecular mobility, and $k T$ the average thermal energy.

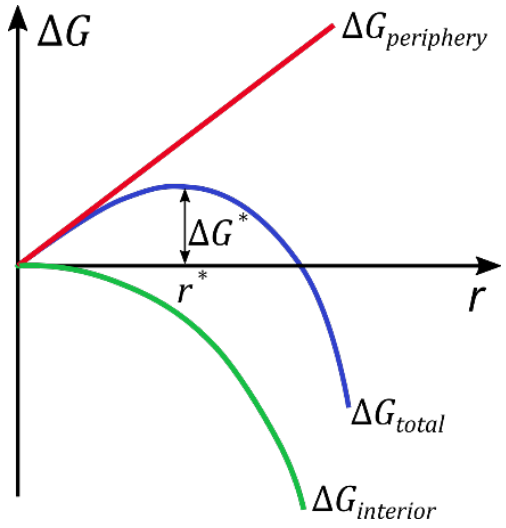

Fig. 1 Gibbs free energy diagram for classical 2D nucleation. The total free energy change (blue) of self-assembly is comprised of an unfavorable term associated with the line tension at the cluster periphery (red), and a favorable term for molecules in the cluster interior (green).

The critical nucleus size is an important parameter in understanding the nucleation mechanism of SAMNs. Unfortunately, little is known about this value due to challenges associated with capturing the stochastic nucleation process at the nanoscale. Using time-resolved atomic force imaging, Sleutel et al. estimated the critical nucleus size of glucose isomerase assemblies on mica to be $\sim 20$ molecules. ${ }^{10}$ More recent work on peptide assemblies on $\mathrm{MoS}_{2}$ showed that nucleation of row-like features can even occur without a free energy barrier. ${ }^{11}$ Nonetheless, for SAMNs formed by small organic molecules, no direct measures of the critical size are available. Given the nanoscale dimensions of nuclei, spatial confinement at this small length scale may provide an advantageous strategy to screen for the critical size.

\section{Nanopatterned surfaces}

Substrates that are naturally or artificially patterned at the nanoscale are ideal platforms to study self-assembly processes under confinement conditions. Interesting patterns include one- and zero-dimensional templates, where molecules are respectively confined along one or both lateral surface directions.

\section{Stepped surfaces}

Step edges act as barriers to adsorbate diffusion, and hence separate surface terraces from their surroundings. On metal surfaces, step edges may by chance be spaced closely together such that confinement effects can be observed on the narrow terraces enclosed. In an early example, Chizhov et al. noticed that the molecular orientation of copper phthalocyanine molecules in a self-assembled network on $\mathrm{Au}(111)$ depends on the terrace width. ${ }^{12}$ On terraces wider than $\sim 15 \mathrm{~nm}$, the molecular orientation is dominated by the substrate lattice symmetry, but on narrower terraces, the sides of the unit cell are aligned along the step edge direction. A similar observation was made by de Oteyza et al. for self-assembled networks of diindenoperylene (DIP) on $\mathrm{Cu}(111)$ as shown in Fig. $2 .{ }^{13}$ On wide terraces, a short range ordered structure is formed with the DIP 


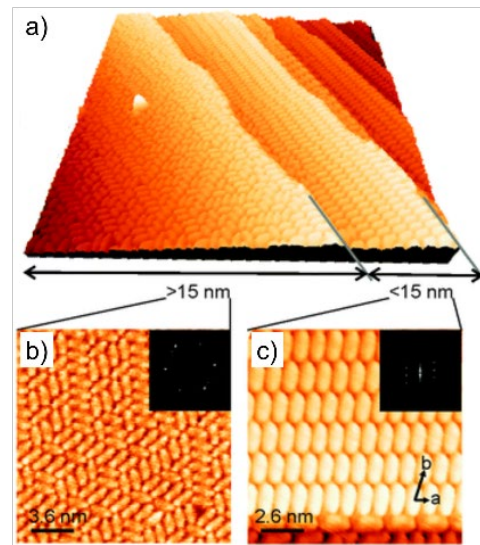

d)
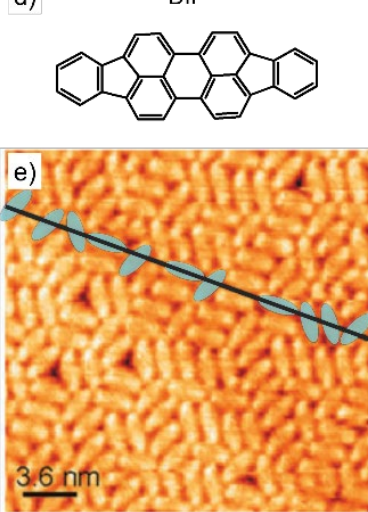

Fig. $253 \times 53 \mathrm{~nm}^{2}$ STM image showing a self-assembled molecular network of DIP on $\mathrm{Cu}(111)$. Two distinctly different structures are observed: (b) a short-range ordered structure on wide terraces, and (c) a long-range ordered structure on terraces narrower than $15 \mathrm{~nm}$. The insets display the corresponding Fourier transforms. (d) Molecular structure of DIP. (e) Illustration of the DIP molecules that would be missing from the short-range ordered structure (blue shaded) in case the black line were a real step edge. Reproduced from ref. 13 with permission from the PCCP Owner Societies.

molecules oriented along three equivalent substrate directions. On the contrary, DIP arranges in a long-range ordered structure with co-directionally oriented molecules on terraces narrower than $15 \mathrm{~nm}$. The altered self-assembly behavior on narrow terraces can be explained on thermodynamic grounds. Since random molecular orientations necessarily leave open spaces in close proximity to the step edges, alignment along the step direction maximizes the enthalpy gain from molecule-substrate interactions by achieving a higher surface coverage. To illustrate the lower coverage in case of random molecular orientations, a fictitious step edge is drawn through the short-range ordered DIP structure in Fig. 2e, highlighting the molecules (blue shaded) that would be missing if the step edge were real. Interestingly, based on the delimiting terrace width of $15 \mathrm{~nm}$, the energy difference between the short- and long-range ordered DIP structure was estimated to be $\geq 0.14 \mathrm{eV}$ per molecule. The actual energy difference, however, will be greater if DIP molecules in the long-range ordered structure are also stabilized by lateral coordination with the step edges.

Previous examples made use of step edges occurring as natural substrate defects. To exploit step edges as well-defined 1D templates, vicinal surfaces of noble metals are particularly interesting. Vicinal surfaces are prepared by cutting the substrate at an angle that slightly deviates from its highsymmetry crystallographic planes, thereby yielding an array of evenly spaced surface steps with adjustable widths. To evaluate the impact of these narrow terraces on SAMN formation, Wang et al. compared self-assembled patterns of 6,13dichloropentacene (DCP, Fig. 3a) on flat $A u(111)$ and stepped $\mathrm{Au}(788) \cdot{ }^{14}$ On flat $\mathrm{Au}(111), \mathrm{DCP}$ monolayers exhibit only small patches of local order with domains oriented along multiple lattice directions (Fig. 3b). In contrast, on the $3.9 \mathrm{~nm}$ wide terraces of $\mathrm{Au}(788), \mathrm{DCP}$ forms a well-ordered, unidirectional brick-wall type network extending over hundreds of nanometers (Fig. 3c). The remarkable improvement of order on
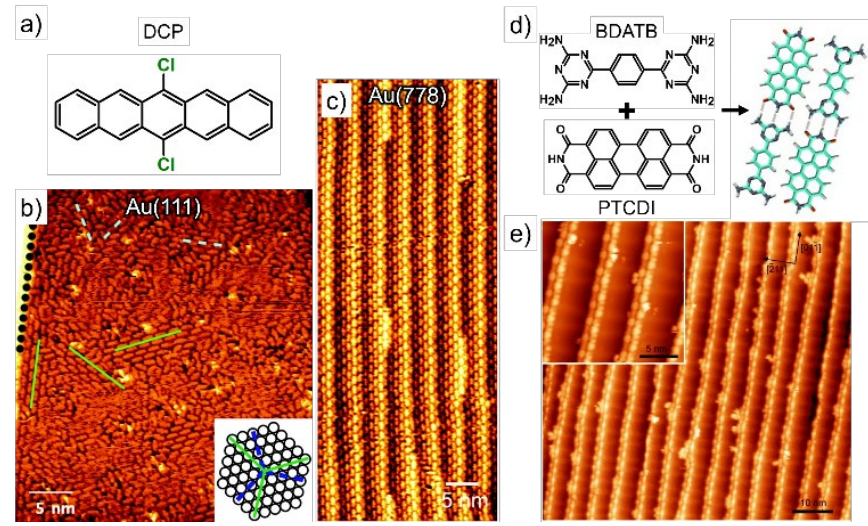

Fig. 3 (a) Molecular structure of DCP. (b) STM image of a DCP network formed on flat $\mathrm{Au}(111)$. Six different molecular orientations are observed: three along the most compact (green) and three along the least compact (dashed blue) lattice directions of the (111) substrate. (c) All DCP molecules are aligned along the step edge direction on $\mathrm{Au}(788)$. Reproduced from ref. 14 with permission from the American Chemical Society, copyright 2011. (d) Molecular structures and bimolecular wire formation from BDATB and PTCDI. (e) STM image showing single and double bimolecular wires running along the lower step edges of $\mathrm{Au}(11,12,12)$. The bright and dark spots correspond to PTCDI and BDATB, respectively. Reproduced from ref. 15 with permission from Wiley-VCH, copyright 2007.

$\mathrm{Au}(788)$ was attributed to an interaction of DCP molecules with the step edges, although the observed unidirectionality in the network might also result from a better surface coverage near the edges as explained above. Similarly, the templating effect of vicinal $\mathrm{Au}(11,12,12)$ has been exploited to fabricate unidirectionally oriented bimolecular wires from 1,4-bis-(2,4diamino-1,3,5-triazine)-benzene (BDATB, Fig. 3d) and 3,4,9,10perylenetetracarboxylic diimide (PTCDI, Fig. $3 d$ ). ${ }^{15}$ As shown in Fig. $3 d$,e, distinct double- or single-row bimolecular wires are formed at low surface coverage, stabilized by three hydrogen bonds on either side of the molecules. The wires consistently run parallel to the lower edges of the surface steps, which suggests that unidirectionality is driven by lateral coordination between the molecular components and substrate atoms at step edges.

Nonetheless, despite the templating and confinement effects described in previous examples, stepped surfaces do not always affect molecular organization. As an example, selfassembly of 3,4,9,10-perylenetetracarboxylic-dianhydride (PTCDA) on $\mathrm{Au}(788)$ or $\mathrm{Au}(778)$ is characterized by domains that seem dominated by intermolecular interactions rather than the template periodicity. ${ }^{16}$ In general, whether stepped surfaces effectively steer supramolecular networks will depend on the balance between interactions within the network and with the substrate, as well as on the ability of molecules to coordinate with substrate atoms at step edges.

\section{Long-range surface reconstructions}

An interesting 1D nanotemplate is the oxygen-induced surface reconstruction of $\mathrm{Cu}(110)$. The $\mathrm{Cu}(110)$ surface consists of rows of tightly packed $\mathrm{Cu}$ atoms oriented along the [1 $1 \overline{1} 0$ ] direction, and as a consequence of its unidirectional corrugation, is 

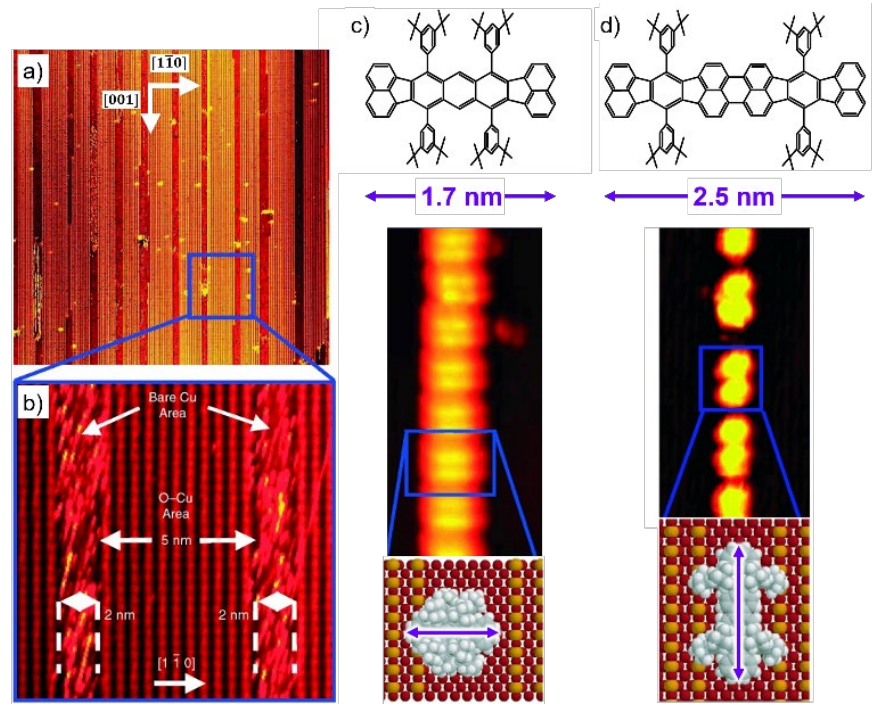

Fig. 4 (a) $70 \times 70 \mathrm{~nm}^{2}$ STM image of the $\mathrm{Cu} / \mathrm{Cu}-\mathrm{O}$ supergrating obtained by exposure of $\mathrm{Cu}(110)$ to oxygen. (b) $14 \times 14 \mathrm{~nm}^{2}$ image showing in detail the bare $\mathrm{Cu}$ and oxygen-passivated stripes running along the [001] substrate lattice direction. The bare $\mathrm{Cu}$ stripes are $2.0 \pm 0.3 \mathrm{~nm}$ wide. (c) Molecular structure of single Lander along with a high-resolution STM image showing the confined self-assembly of SL on the bare Cu stripes. From the STM image,

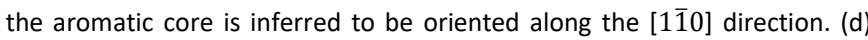
Molecular structure and confined self-assembly of violet Lander. Due to their longer length, VL molecules are oriented along the [001] substrate direction. Reproduced from ref. 7 with permission from Wiley-VCH, copyright 2004.

expected to align rod-shaped molecules in this direction. Exposure of this surface to oxygen under high pressure and temperature in UHV leads to rows of chemisorbed oxygen running along the [001] direction. Under appropriate exposure conditions, the reconstruction is only partial, and a periodic supergrating of alternating bare $\mathrm{Cu}(110)$ and oxygen-passivated $(2 \times 1) \mathrm{Cu}-\mathrm{O}$ stripes with controllable dimensions is formed as shown in Fig. 4a,b. This 1D template represents a useful platform to study molecular organization in confined spaces since organic molecules will preferentially adsorb on the more polarizable bare $\mathrm{Cu}(110)$ stripes.

Confinement effects arising from adsorption on a $\mathrm{Cu} / \mathrm{Cu}-\mathrm{O}$ template were shown for so-called "Lander molecules". ${ }^{7}$ Both single (SL) and violet (VL) Lander molecules consist of an aromatic core substituted with four 3,5-tert-butylphenyl spacer legs as shown in Fig. 4c,d. When deposited on a periodic $\mathrm{Cu} / \mathrm{Cu}$ $\mathrm{O}$ nanotemplate, $\mathrm{SL}$ and $\mathrm{VL}$ adsorb exclusively on the bare $\mathrm{Cu}$ stripes, albeit with a different orientation (Fig. 4c,d). For SL, the aromatic core is oriented along the [1 $1 \overline{1} 0]$ direction as it would also on pristine $\mathrm{Cu}(110)$. This result is expected since the core length $(\sim 1.7 \mathrm{~nm})$ fits well onto the $\mathrm{Cu}$ stripes, and hence no reason for an altered adsorption orientation exists. The situation is quite different though for the longer Violet Lander (VL) molecule. Due to its extended core length $(\sim 2.5 \mathrm{~nm})$,

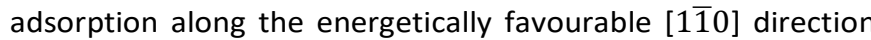
would imply that the molecules are partially adsorbed on top of the $\mathrm{Cu}-\mathrm{O}$ regions. To avoid adsorption on the oxygen-passivated regions, the $\mathrm{VL}$ molecules consequently reorient on the $\mathrm{Cu} / \mathrm{Cu}$ $\mathrm{O}$ template such that their cores are aligned closely to the $\mathrm{Cu}-\mathrm{O}$ stripes (i.e. the [001] direction). Such molecular reorientation is expected to take place whenever the molecular length approaches or exceeds the template dimensions. Besides the molecular orientation, nanoscopic confinement on a $\mathrm{Cu} / \mathrm{Cu}-\mathrm{O}$ supergrating can also impact the supramolecular packing arrangement. For instance, Cicoira et al. showed that rubrene SAMNs on 5-10 nm wide Cu stripes are more dense compared to their analogues formed on pristine surfaces. ${ }^{17}$

\section{Etch pits}

In 1990, Chang and Bard reported the formation of monolayer deep pits on the basal plane of HOPG when heated in air at 650 ${ }^{\circ} \mathrm{C} .{ }^{18}$ These pits are formed by oxidation at intrinsic defects in the graphite substrate, and are mostly circular in shape with a diameter that scales linearly with etch time. Given that the underlying graphite layer remains intact, the resulting pits are particularly interesting as $\mathrm{OD}$ templates to isolate ensembles of molecules and study their confined self-assembly behavior. When used for this purpose, these etch pits are typically referred to as "molecule corrals" in the literature in analogy with the enclosures used to capture livestock.

Beebe and coworkers used these molecule corrals to study the impact of confinement on the molecular ordering behavior of the liquid crystalline compound 4'-octyl-4biphenylcarbonitrile ( $8 \mathrm{CB}$, Fig. $5 a) .{ }^{8}$ Fig. 5 b shows an STM image of the interface between an $8 C B$ film and a graphite surface patterned with molecule corrals. On the surrounding terrace, self-assembled rows are seen that are ascribed to a head-tohead configuration of $8 \mathrm{CB}$ molecules. The image also shows two molecule corrals; one filled with an ordered $8 \mathrm{CB}$ domain, and one which appears empty. Most likely, the "empty" corral is filled with disordered molecules that cannot be resolved on the time-scale of STM imaging. Over time, the empty corrals also get filled with ordered assemblies. Since nucleation is typically much slower than domain growth, the rate of the disorder-toorder transition essentially equals the nucleation rate. Hence,
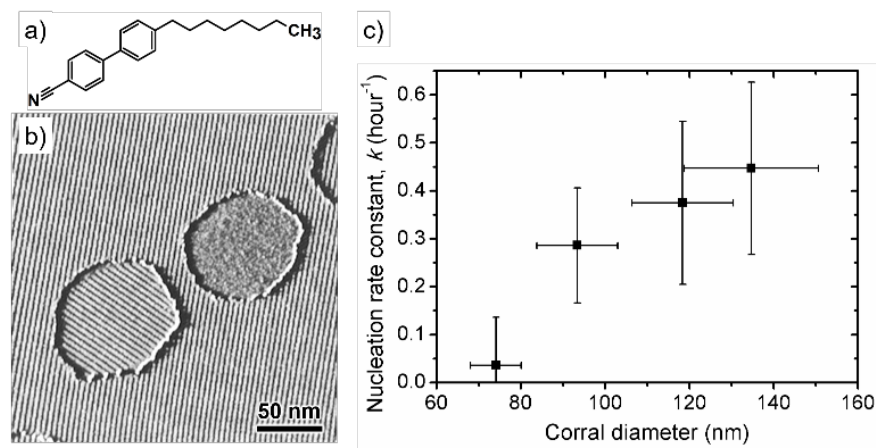

Fig. 5 (a) Molecular structure of the liquid crystalline compound 8CB. (b) STM current image of $8 C B$ self-assembly on graphite patterned with molecular corrals. Row-like features indicative of an ordered $8 \mathrm{CB}$ network are visible inside the lower left corral, as well as on the surrounding terrace. No ordered structures are seen for the corral on the right. (c) Measured nucleation rate constants for different corral sizes. Reproduced from ref. 19 with permission from the American Chemical Society, copyright 1996. Graph based on data in ref. 8 with permission from the American Association for the Advancement of Science, copyright 1994. 


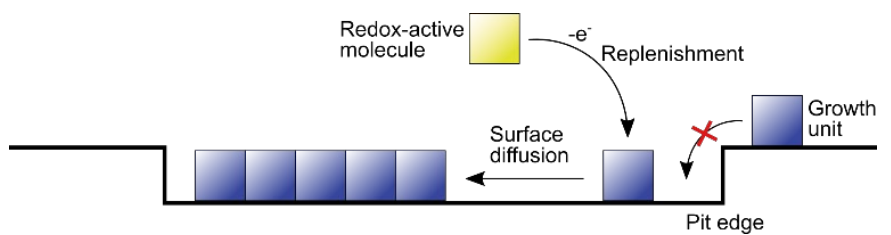

Fig. 6 Limited transport of growth units to the corral interior due to pit edge discontinuities. Adapted from ref. 20 with permission from the American Chemical Society, copyright 1998.

by examining the fraction of corrals containing ordered assemblies as a function of time, it is possible to measure the nucleation rate for different sized corrals. As shown in Fig. 5c, the nucleation rate constant decreases with decreasing corral size. Furthermore, analysis of corrals with different shapes and sizes reveals that large corral perimeter/area ratios negatively impact the nucleation rate. This implies that corral edges inhibit nucleation, and that nucleation preferentially occurs at open terraces away from any step edges. The inhibitory effect of corral edges on nucleation is attributed to a perturbation of molecular ordering in their vicinity, such that nuclei forming near an edge are less likely to attain their critical size. In addition, temperature-dependent studies reveal that the nucleation rate constant increases sharply with decreasing temperature. ${ }^{19}$ This behavior qualitatively and quantitatively agrees with CNT, and seems to confirm that nucleation inside the corrals can be interpreted by the classical model, where nucleation is the rate-limiting step in the formation of ordered $8 \mathrm{CB}$ assemblies, and where each corral is characterized by a distinct nucleation event.

In previous account, $8 \mathrm{CB}$ was applied as a neat liquid to graphite substrates patterned with molecule corrals. As such, adsorption of a disordered monolayer on the substrate is virtually instantaneous, and the formation of ordered assemblies is inherently a 2D order-to-disorder transition. In contrast thereto, Hooks et al. examined the nanoconfined electrochemical nucleation

of bis(ethylenedithiolo)tetrathiafulvalene triiodide, $(E T)_{2} l_{3}$, from dilute acetonitrile solutions. ${ }^{20}$ Upon application of the growth potential, $(E T)_{2} l_{3}$ can form two different monolayer structures on graphite, referred to as type I and type II. In a similar fashion to the $8 \mathrm{CB}$ study, the nucleation rate of $(\mathrm{ET})_{2} \mathrm{l}_{3}$ was measured by studying the fraction of filled corral over time. For both type I and type II structures, nucleation is slower in smaller corrals, and no assemblies are observed to form inside corrals with a radius below $50 \mathrm{~nm}$. Furthermore, the nucleation rate of type II monolayers, which exhibit only half the packing density of type I monolayers, is approximately twice that of type I monolayers. While the suppression of nucleation in corrals can again be explained by the difficulty of nuclei to attain their critical size in confined spaces, the inverse correlation between nucleation rate and packing density suggests that nucleation is also limited by transport of molecular growth units to growing nuclei. The limited transport of growth units to corrals can be understood by considering the fact that corral edges form a barrier towards surface diffusion. As such, the supply of growth units to the corral interior relies mainly on direct adsorption from solution
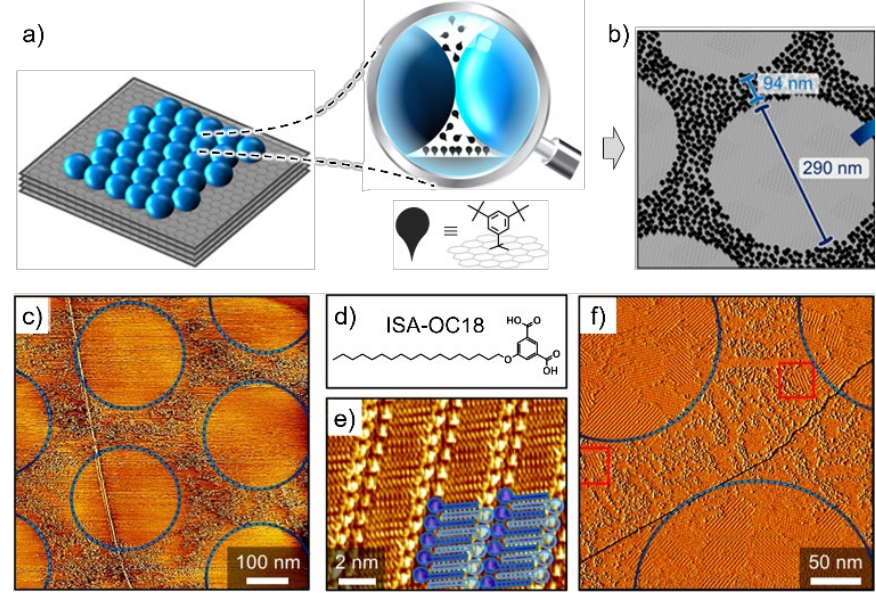

Fig. 7 (a) Hexagonal polystyrene bead template for patterned covalent surface modification. (b) Patterned graphite surface after removal of the bead template. (c) STM image of the periodically arranged corrals obtained after electrochemical grafting and template removal. (d) Molecular structure of ISA-OC18. (e) High-resolution STM image of a self-assembled ISA-OC18 network at the octanoic acid/HOPG interface. (f) STM current image of confined ISA-OC18 networks assembled from octanoic acid in circular nanocorrals. Occasionally, regions of lower grafting density trap small domains of self-assembled ISA-OC18 molecules (red boxes). Reproduced from ref. 23 with permission from the American Chemical Society, copyright 2018.

as schematically illustrated in Fig. 6. The importance of efficient molecular transport is expected to increase for lower solute concentrations.

Besides confining molecules in their interior, etch pits on graphite can also act as controllable surface defects towards molecules adsorbed on the upper terrace surrounding the corrals. In that case, the mean defect-free distance, determined by the corral density, defines the degree of confinement experienced by the self-assembling molecules. For instance, Tracz et al. found that no ordered assemblies of alkylated oligothiophene derivatives could form when the mean defectfree distance was on the order of two to three times the width of a molecular lamella. ${ }^{21}$ From this observation, the critical nucleus size was inferred to be of similar dimensions. Furthermore, when sufficiently close together, the corrals can pin (i.e. hold in place) domain boundaries between different monolayer structures in a metastable arrangement. ${ }^{22}$

\section{Grafted templates}

Besides oxidative etching, our lab recently suggested covalent surface modification of graphite as an alternative strategy to generate a variety of patterns that can be of interest to study confined molecular self-assembly. ${ }^{9}$ The modification is based on the reduction of aryl diazonium salts under electrochemical control, followed by covalent binding of the generated aryl radicals to the graphite surface. Rational choice of the experimental conditions and/or diazonium precursors allows to regulate the density of grafted aryls from sparsely distributed grafts to a dense monolayer.

Under conditions where a dense monolayer of aryl species is grafted, the surface is completely blocked for self-assembly. However, by using a self-assembled network of polystryrene 

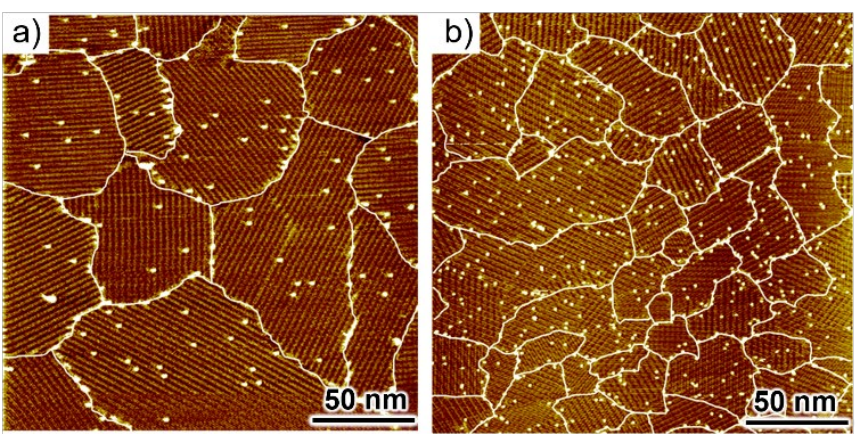

c)

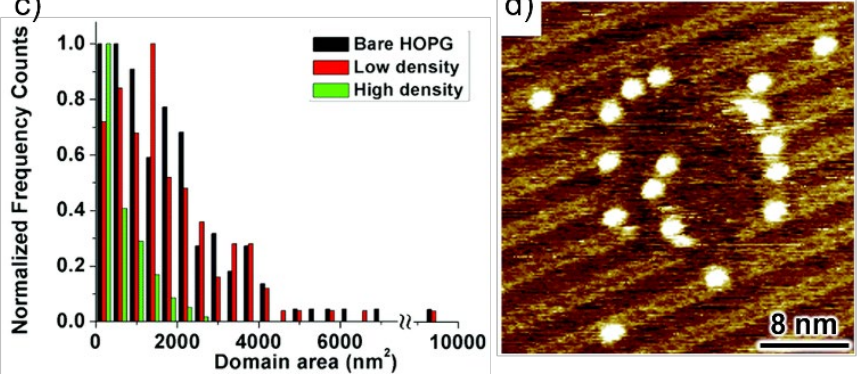

Fig. 8 STM image of self-assembled ISA-OC18 domains from octanoic acid solution on (a) low density, and (b) high density grafted graphite substrates. The grafted species correspond to the bright dots in the image. (c) Histogram of the domain size distribution on bare HOPG, as well as on low and high density grafted HOPG. (d) STM image showing the absence of ordered ISAOC18 structures in regions of high confinement. Reproduced from ref. 25 with permission from the Royal Society of Chemistry, copyright 2016.

beads as a lithographic mask, electrochemical grafting can be directed exclusively to the area in between the beads (Fig. 7a,b) Subsequent removal of the beads leads to a hexagonal array of circular patterns of pristine graphite that can be used as molecule corrals (Fig. 7c). ${ }^{23}$ Self-assembly of 5-octadecyloxyisophthalic acid from solution (ISA-OC18, Fig. 7d) inside these circular corrals results in the formation of many small domains per corral (Fig. 7f), with an average size being nearly twenty times smaller than domains formed on bare graphite substrates. The significant reduction of domain sizes inside the corrals was suggested to be indicative of hindered Ostwald ripening in the confined spaces, although the impact of potential polystyrene residues was not ruled out. Recently, we demonstrated a mask-free strategy for creating circular corrals on graphite, thus avoiding any residues. ${ }^{24}$ This novel strategy is based on electrochemical grafting from a mixture of aryl diazonium salts, which surprisingly leads to the spontaneous formation of circular patterns.

At lower grafting density, the grafts do not completely block the surface, but instead act as randomly distributed surface defects, not unlike the impact of etch pits on molecules adsorbed on the surrounding terraces. Self-assembled domains of ISA-OC18 on such modified substrates exhibit an inverse correlation between domain size and grafting density as shown in Fig. 8a-c, which was attributed to hindered domain growth. ${ }^{25}$ In cases of extreme confinement due to a dense agglomeration of grafted defects, often no ordered assemblies are formed ( Fig. 8d). The lack of ordered structures in these regions may indicate that the critical nucleus size cannot be attained. In another study, the randomly dispersed grafts were shown to alter the packing morphology of a dehydrobenzo[12]annulene derivative bearing six tridecyloxy chains (DBA-OC13). ${ }^{26}$ Whereas a porous network consisting of $C_{6}$-symmetric pores is favored on bare graphite, the introduction of grafted defects results in less symmetric pores and non-porous packing arrangements. These different structures are believed to be pinned in a metastable situation by the grafts.

\section{Scanning probe lithography}

The ability of scanning probe microscopes to manipulate atoms or molecules on a surface has been of great interest for the creation of nanoscale patterns. When used in this mode, the technique is commonly referred to as scanning probe lithography (SPL). While many variations of SPL exist, in its most basic form, the probe is used to remove molecules locally from a surface, often referred to as nanoshaving. The "void" spaces thus generated can subsequently be filled with other molecular adsorbates resulting in a net substitution. This type of substitution lithography offers exciting possibilities to explore confinement effects on 2D supramolecular self-assembly in well-defined patterns.

\section{Nanografting}

Liu and coworkers reported a nanofabrication method named nanografting, which effectively was the first demonstration of in situ substitution SPL. ${ }^{27,} 28$ In their method, an AFM tip is used to perform nanoshaving on a self-assembled thiolate matrix on $\mathrm{Au}(111)$ that is immersed in a solution containing thiol molecules different from the matrix. As the tip shaves through the matrix, thiolate molecules from the matrix are expelled, and molecules from solution adsorb and self-assemble in the void spaces as illustrated in Fig. 9b. While the mechanisms constituting alkyl thiolate monolayer formation on gold are markedly different from the formation of physisorbed SAMNs, several interesting confinement effects were nevertheless observed.

Firstly, the kinetics of self-assembly inside the void spaces are at least one order of magnitude faster than those on bare gold substrates. ${ }^{29}$ This remarkable acceleration points out a change in the assembly pathway effectuated by the spatially constrained environment during nanografting. During nanografting, adsorption of thiol molecules in the void spaces is sterically hindered by the surrounding matrix as well as the bulky AFM tip. Consequently, it was suggested that thiol molecules adsorbing in this nanoenvironment bypass the lyingdown phase that is typical for self-assembly on bare substrates, and instead adopt the standing-up configuration immediately (Fig. 9a,b). In the standing-up configuration, the chains are stabilized by van der Waals contact with the surrounding matrix, which lowers the activation energy for binding of sulphur to the gold substrate. Therefore, spatial confinement can effectively accelerate the assembly process.

Secondly, the structure and composition of nanografted binary thiolate monolayers is significantly more homogeneous compared to naturally-grown layers, with the degree of 

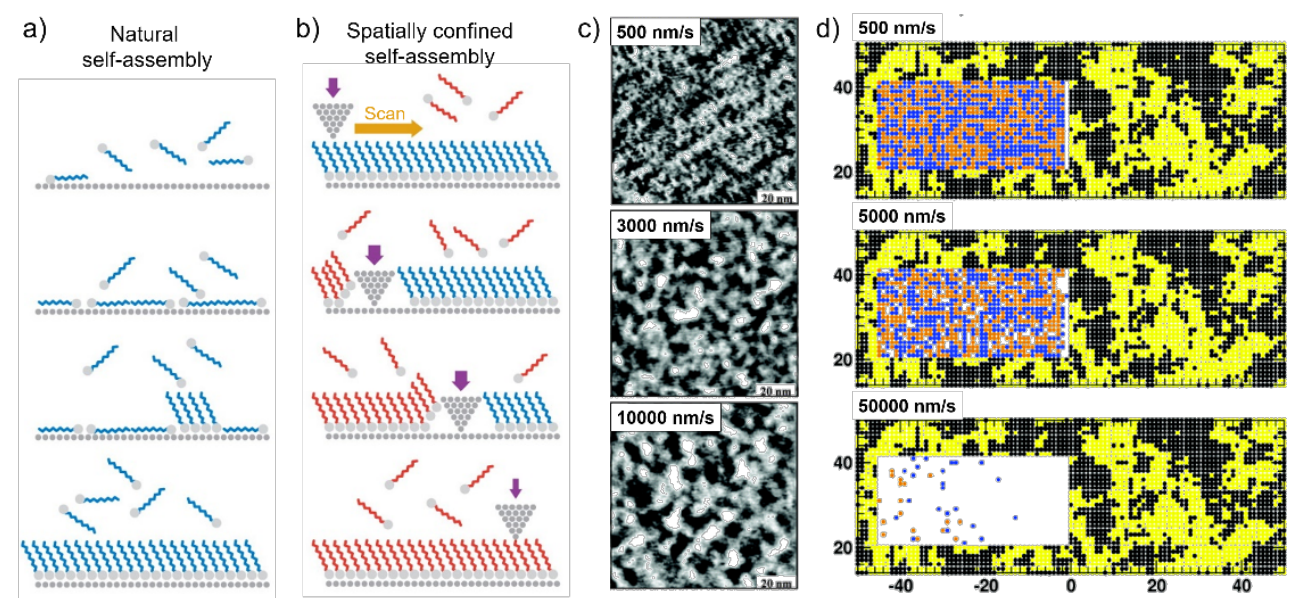

Fig. 9 (a) Natural self-assembly of alkyl thiolate monolayers on gold. Alkyl thiols initially adsorb in a lying-down configuration, followed by a transition to the chemisorbed standing-up configuration. (b) Spatially confined self-assembly during nanografting. In the restricted environment behind the probe, alkyl thiol molecules (red) immediately adopt the standing-up configuration. Reproduced from ref. 28 with permission from Annual Reviews, copyright 2008. (c) AFM topography images of binary self-assembled monolayers obtained by nanografting at different shaving speeds. Nanografting was performed in a 2butanol solution with $\mathrm{C}_{18} \mathrm{SH}: \mathrm{C}_{10} \mathrm{SH}=1: 5$, and a total concentration of $0.02 \mathrm{mM}$. The slower the shaving speed, the more homogeneous the monolayer. Reproduced from ref. 30 with permission from the American Chemical Society, copyright 2006. (d) Monte Carlo simulation of the nanografting dynamics for a 1:1 binary mixture of $\mathrm{C}_{18} \mathrm{SH}$ and $\mathrm{C}_{10} \mathrm{SH}$. A single line having width of 21 lattice positions ( $y$-axis) is being shaved along the $x$-axis at various tip speeds. Each dot represents a lattice site that can be occupied by $\mathrm{C}_{18} \mathrm{SH}$ (yellow for matrix, orange for nanografted area) or $\mathrm{C}_{10} \mathrm{SH}$ (black for matrix, blue for nanografted area). Reproduced from ref. 31 with permission from the American Chemical Society, copyright 2006.

homogeneity decreasing at faster shaving speeds. ${ }^{30}$ The formation of a more homogeneous film is a direct consequence of the above-mentioned altered assembly pathway. In the confined environment during nanografting, little space and time is available for on-surface diffusion. Therefore, adsorption events dominate and self-assembled structures are under kinetic control. The faster the shaving speed, the more space and time is allowed for the formation phase-segregated domains leading again to more heterogeneous monolayers (Fig. 9c). The dynamics of self-assembly during nanografting were accurately described by the kinetic Monte Carlo model developed by Ryu and Schatz. ${ }^{31}$ As shown in Fig. 9d, at the slowest shaving speed, a complete monolayer is quickly formed behind the tip as adsorption in the standing-up conformation is sterically favored. At intermediate shaving speed, the monolayer is incomplete and part of the molecules are in their physisorbed state such that they can keep diffusing to form phase-segregated domains. At fast shaving speed, the tip leaves behind a large open space, where molecules adsorb in their physisorbed lying-down state with ample time and space for onsurface diffusion.

Note that the shaving speed becomes an important parameter whenever confinement effects from in situ substitution lithography are interpreted: since adsorption concurs with shaving, the voids to be filled are transient, and the steric constraints for adsorption and self-assembly are determined by the shaving speed.

\section{Nanoshaving on a phyisorbed template}

In analogy with the nanografting technique, in situ substitution SPL can also be used to study the impact of (transient) confinement on the formation of physisorbed SAMNs. Using a self-assembled matrix of perylene-3,4,9,10-tetracarboxylicdianhydride (PTCDA, Fig. 10a) on graphite, Isoda and coworkers investigated the confined self-assembly of 17,19dotetracontadiyne (DTDY, Fig. 10a). ${ }^{32}$ STM was used to remove the matrix molecules and create void spaces for self-assembly. Under these conditions, DTDY molecules preferentially adsorb in the void spaces with their molecular axis aligned along the high-symmetry axis of the substrate nearest to the fast scan axis a)

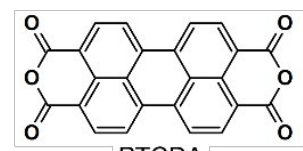
PTCDA
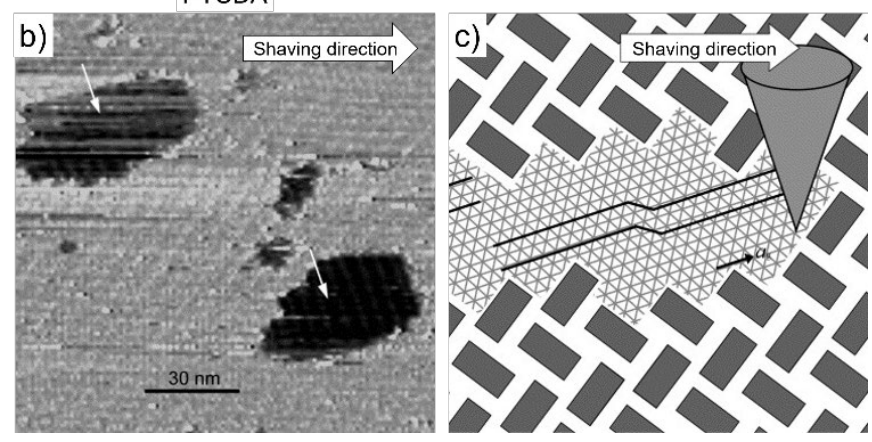

Fig. 10 (a) Molecular structure of PTCDA and DTDY. (b) STM image of two self-assembled DTDY domains within a PTCDA matrix created by in situ nanoshaving at the 1-phenyloctane/HOPG interface. The white arrows indicate the orientation of the DTDY columns. (c) Schematic illustration of the proposed alignment mechanism. The narrow space created along the track of the STM tip fits only DTDY molecules that are aligned along the shaving direction. Adapted from ref. 32 with permission from Elsevier Science B.V., copyright 2003. 
a)

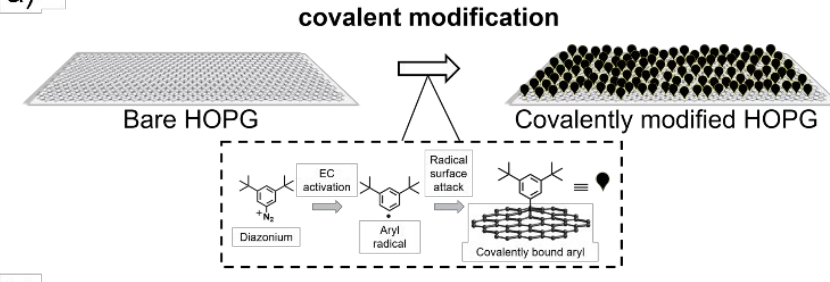

b)

c)

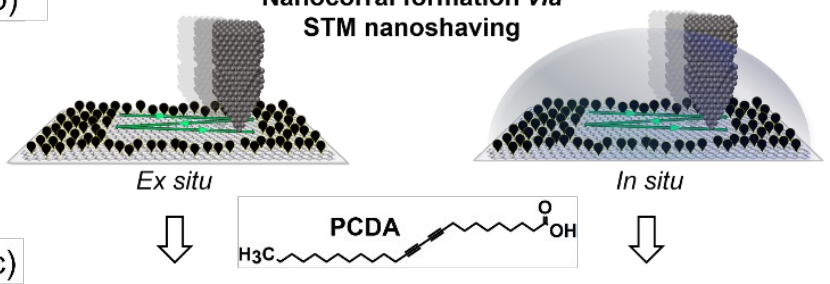

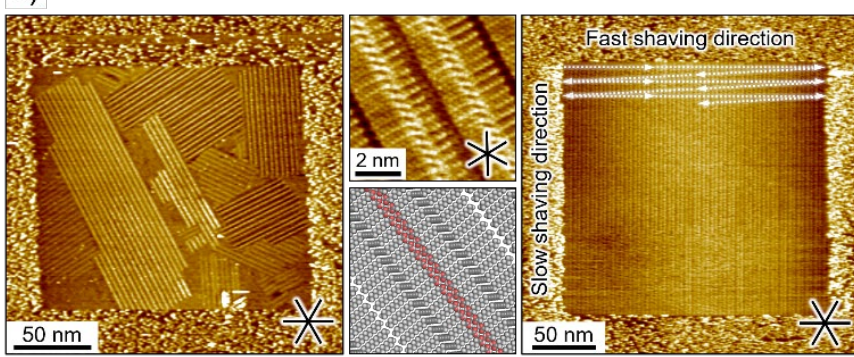

Fig. 11 Schematic illustration of (a) covalent surface modification of HOPG, and (b) nanoshaving (ex situ and in situ) performed by the STM tip. (c) STM images of the confined PCDA assemblies at the 1-phenyloctane/HOPG interface. Whereas, multiple rotational domains are present in the ex situ fabricated corral, only a single domain with the molecules aligned along the fast shaving direction (horizontal axis) is observed in the in situ corral. High resolution STM image and molecular model of PCDA assembly shown in the middle. Reproduced from ref. 34 with permission from the American Chemical Society, copyright 2016.

as shown in Fig. 10b. This alignment effect is believed to be a consequence of the transient spatial constraints emerging from the line-by-line removal of matrix molecules. In the resulting narrow spaces created along the track of the tip, the long-chain molecules are sterically forced to adsorb in the orientation closest to the shaving direction as shown in Fig. 10c.

In a following study, the authors exploited the spatial constraints emerging from the in situ removal of a PTCDA matrix to achieve selective molecular adsorption form a mixed solution of 10,12-pentacosadiynoic acid (PCDA) and 3-imino-4,5,6,7tetrachloroisoindolin-1-one (ITCII). ${ }^{33}$ As ITCII molecules are smaller than PCDA molecules, they fit the void spaces along the track of the tip before the PCDA molecules do. Consequently, the nanoshaved areas are consistently filled with ITCII domains, even under conditions favoring adsorption of PCDA on bare HOPG.

\section{Nanoshaving on a chemisorbed template}

A particular drawback of using a physisorbed molecular network as the matrix for substitution lithography is the limited stability of molecules at the edge of a nanoshaved region, leading to larger and more irregular patterns than intended. To avoid this problem, more rigid matrices made of chemisorbed species can a)

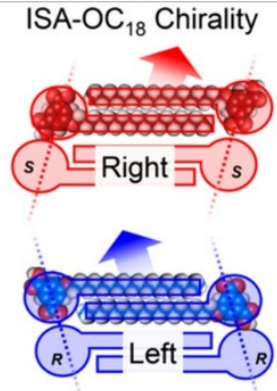

b) Surface Lattice Relationships

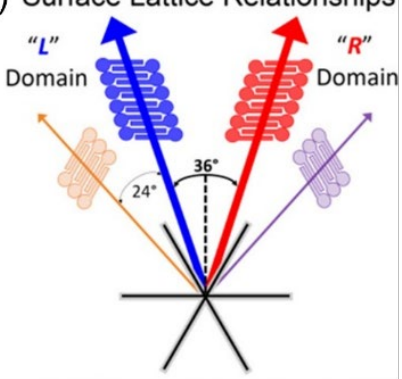

"L" Chiral Square

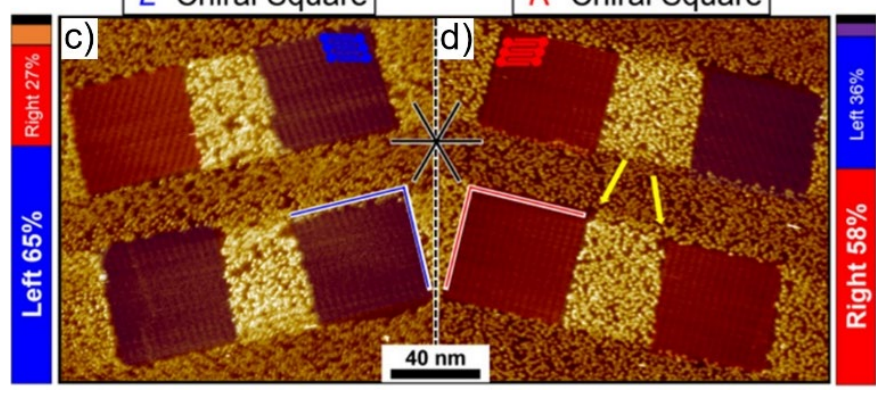

Fig. 12 (a) Schematic illustration of the chirality of ISA-OC18 domains selfassembled at the octanoic acid/HOPG interface. Molecules with $S$ and $R$ chirality stack in right and left tilted lamellar enantiomorphs, respectively. (b) Illustration of the registry between ISA-OC18 domains and the graphite substrate (high-symmetry axes shown in black). (c,d) STM images of selfassembled ISA-OC18 domains formed inside left and right chiral square nanocorrals created in situ at the octanoic acid/HOPG interface. Statistics on the domain frequencies are shown on the side of the STM images. Colors refer to the domains illustrated in (b). Reproduced from ref. 37 with permission from the American Chemical Society, copyright 2018.

be used. As mentioned above, a rigid matrix can be obtained from the electrochemical surface modification of graphite using aryl diazonium salts. Using bulky substituents on the aryl diazonium precursors in combination with a high concentration, a densely grafted monolayer capable of blocking the graphite surface is obtained (Fig. 11a). ${ }^{9}$ The grafts can, however, easily be removed by STM nanoshaving, offering the possibility to create well-defined and stable patterns that can be used as molecule corrals (Fig. 11b). In contrast to the work of Isoda et al., the improved stability of the patterns thus created enables the fabrication of corrals prior to addition of a solution (referred to as ex situ nanoshaving), in addition to in situ nanoshaving at the liquid-solid interface. ${ }^{34}$ Comparing both shaving methods in Fig. 11c, it is clear that PCDA assemblies are rather unaffected by confinement in ex situ created corrals, whereas they exhibit a strong tendency to align with their molecular axis as close as allowed by substrate registry to the shaving direction in in situ created corrals. Given that alignment is only observed for in situ nanoshaving, the preferential molecular orientation can be attributed to transient constraints during the shaving process similar to Fig. 10c. In that case, the degree of alignment is expected to decrease at faster shaving speed as this would allow more time and space for self-assembly in analogy with the nanografting work described above. Experimentally, however, the degree of alignment increases with shaving speed, which 
a)
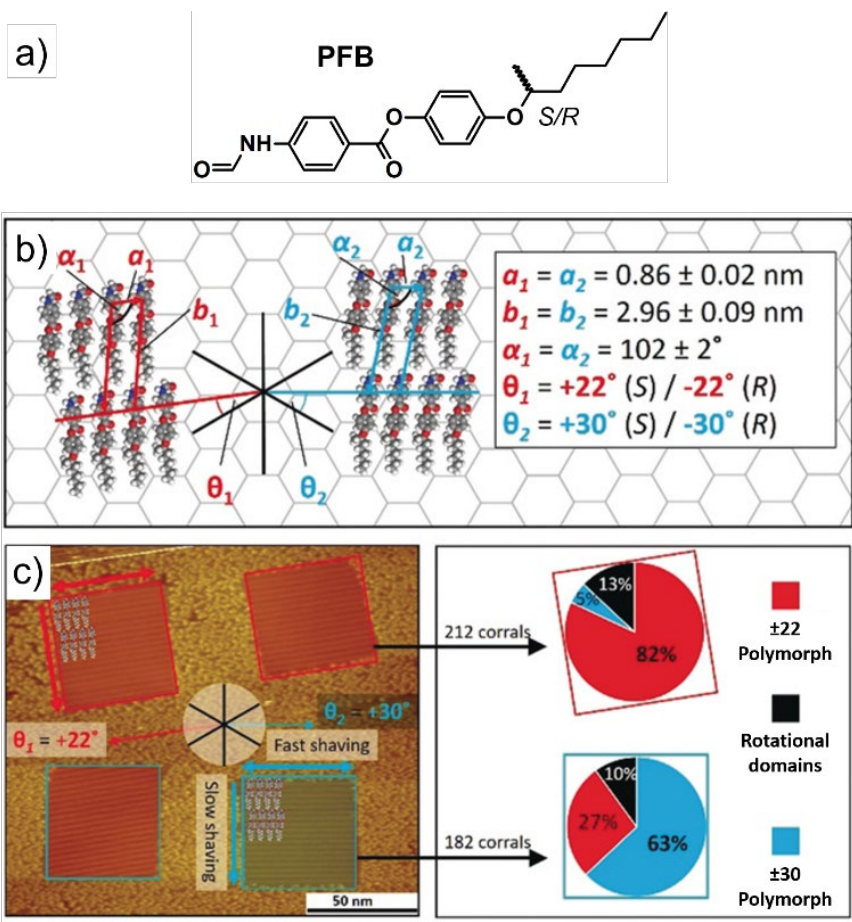

Fig. 13 (a) Molecular structure of PFB. (b) Tentative model of the two lamellar polymorphs formed by S-PFB at the 1-phenyloctane/HOPG interface. (c) STM image showing S-PFB self-assembled in in situ created nanocorrals differing in their orientation relative to the underlying graphite lattice, along with the obtained statistics. Reproduced from ref. 38 with permission from Wiley-VCH, copyright 2019.

seems to suggest that the scanning action of the tip, rather than spatial constraints, assists in orienting the molecules during shaving. ${ }^{35}$ Such tip-induced alignment of alkylated molecules has indeed been observed on several occasions. ${ }^{36}$

Given the ability to select rotational domains using in situ STM nanoshaving on a grafted matrix, the possibility to also select chiral domains was subsequently explored. ${ }^{37} \mathrm{~A}$ prochiral molecule, ISA-OC18, was chosen for its self-assembly in chiral domains consisting of left- or right-handed lamellar enantiomorphs (Fig. 12a,b). To preferentially bias the domain chirality, square nanocorrals were created at an oblique angle with respect to the underlying substrate, such that the corral borders are tilted at an angle corresponding to the ISA-OC18 lamellae orientations. As shown in Fig. $12 \mathrm{c}, \mathrm{d}$, under these conditions, ISA-OC18 molecules tend to form domains of matching handedness in the respective corrals with roughly a two to one preference ratio. This bias in the enantiomorph formation is attributed to a better filling of the available corral spaces (i.e. a higher surface coverage) for domains of matching handedness, hence leading to a reduction of the free energy. Importantly, improved stability from better space filling may act on emerging nuclei during the initial stages of corral formation, as well as on mature domains in completed corrals.

Similar to the strategy described for biasing the formation of enantiomorphs, careful choice of shaving orientation with respect to the substrate lattice can be used to select specific polymorphs. ${ }^{38}$ This effect was demonstrated for $4-[(S / R-1-$ methylheptyl)oxy]phenyl-4'-formamidobenzoate

$(S / R-P F B)$
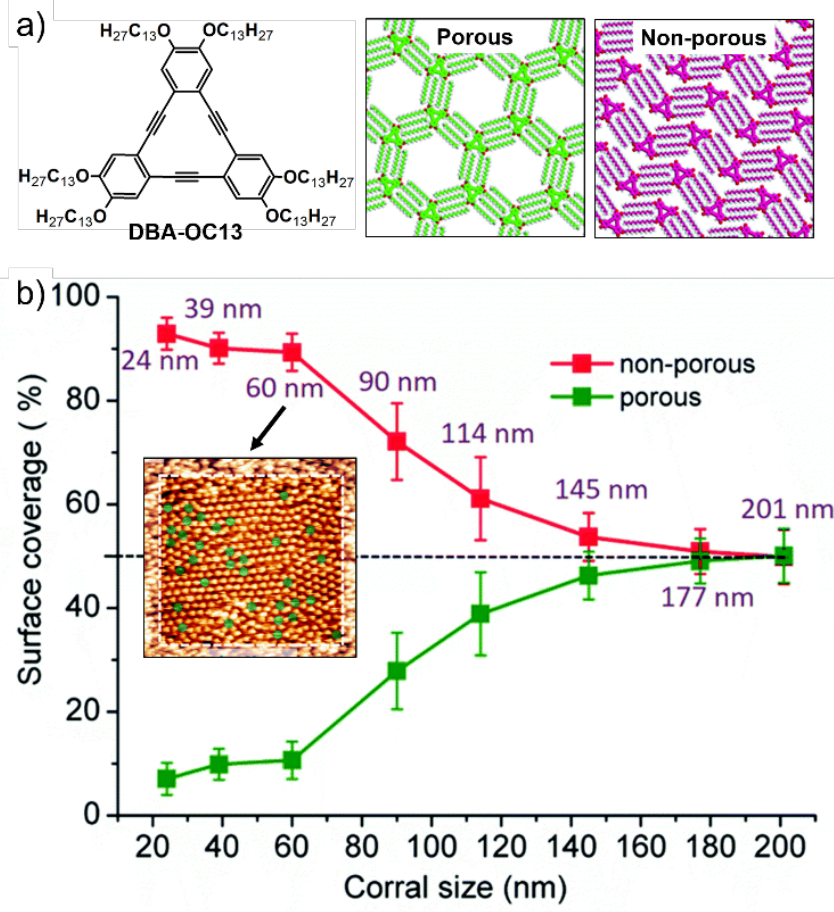

Fig. 14 (a) Molecular structure of DBA-OC13 along with a tentative model of the porous and non-porous phases. (b) Surface coverage of the porous and non-porous phase as a function of corral size. The inset shows an STM image of a $60 \times 60 \mathrm{~nm}^{2}$ corral obtained at the 1-phenyloctane/HOPG interface. The pores of the porous phase are highlighted in green. Reproduced from ref. 39 with permission from the Royal Society of Chemistry, copyright 2019.

which forms two lamellar polymorphs characterized by a different relative orientation with respect to the graphite substrate (Fig. 13a,b). Upon in situ nanoshaving, the lamellar polymorph that runs most closely to the corral borders is formed preferentially as shown in Fig. 13c. Interestingly, the induced bias is highest for the minority polymorph on bare graphite. Such a bias can again be explained on the basis of optimal space filling by the polymorph that runs parallel to the corral borders.

For DBA-OC13, a compound that self-assembles in both a porous and non-porous structure at the 1-phenyloctane/HOPG interface (Fig. 14a), the corral dimensions rather than their orientation impacts the expression of polymorphism. ${ }^{39}$ At a solute concentration yielding similar surface coverage of both structure on bare graphite, confinement inside in situ created corrals tends to shift the balance in favor of the denser, nonporous structure. As shown in Fig. 14b, the prevalence of the non-porous structure increases gradually for smaller corral dimensions, which suggests a true confinement effect. On the one hand, the preference for the non-porous structure may reflect a higher nucleation probability of this phase owing to potentially smaller dimensions of its critical nucleus. On the other hand, interactions with the corral walls might stabilize the denser phase thermodynamically.

Finally, confinement inside nanoshaved corrals can suppress long-range order of a self-assembled network due to geometric frustration. ${ }^{40}$ Such frustration arises from a mismatch between 


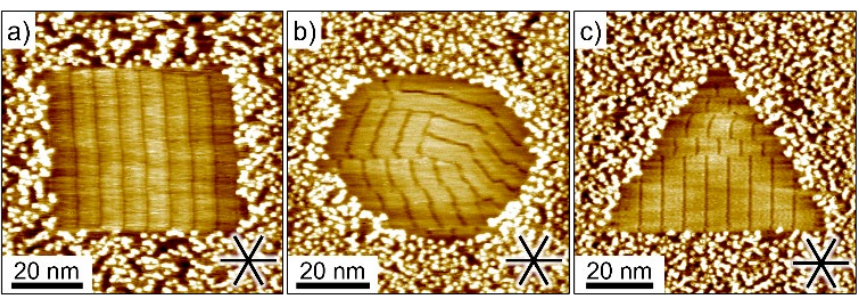

Fig. 15 STM images showing the impact of geometric frustration on the assembly of $n-\mathrm{C}_{50} \mathrm{H}_{102}$ inside various nanocorrals at the 1-phenyloctane/HOPG interface. (a) Square nanocorrals foster linear lamellae. (b,c) Geometric frustration inside circular and triangular nanocorrals induces deformed and defected lamellae. Reproduced from ref. 40 with permission from the American Chemical Society, copyright 2019.

the network morphology and the corral shape. For instance, the linear lamellae of $n-\mathrm{C}_{50} \mathrm{H}_{102}$ pack well within square-shaped corrals, but deform inside circular or triangular corrals as illustrated in Fig. 15. This deformation, and concurrent loss of order, reflects a tendency for optimal space filling in order to maximize the free energy gain from molecule-substrate interactions in spite of molecule-molecule interactions. Such behavior is thought to be a general phenomenon for systems stabilized by weak, non-directional intermolecular forces.

\section{Reactivity under confinement}

Reactions between molecules that are adsorbed or preorganized on a surface are being widely explored as a route to create new and robust nanomaterials. A key goal in this emerging field is to obtain precise control over the structure and morphology of the reaction products. In this regard, the use of surface templates that spatially confine the reactants has been recognized as a promising approach to steer the outcome for a variety of reactions in different environments.

\section{D templates}

The long-range, anisotropic morphology of stepped surfaces is well-suited to align reaction products and facilitate the formation of 1D molecular chains. This effect is clearly illustrated by comparing the alkyne homocoupling between 4,4'"-diethynyl-1,1':4',1'terphenyl (DETP, Fig. 16a) molecules on a planar $\mathrm{Ag}(111)$ versus a vicinal $\mathrm{Ag}(877)$ surface. ${ }^{41}$ On $\mathrm{Ag}(111)$, thermal annealing at $400 \mathrm{~K}$ induces a variety of reactions as shown in Fig. 16b. Linear structures are observed in roughly one out of three cases, the rest being a mixture of branched products. In contrast thereto, linear polymers aligned along the substrate step edges are the main product formed on $\mathrm{Ag}(877)$ (Fig. 16c). The selectivity for linear products on the vicinal surface is predetermined by the organization of DETP precursor molecules parallel to the step edges, which is believed to persist at elevated temperatures. Besides improving the chemoselectivity and aligning reaction products, step edges on metal surfaces may also act as catalytic sites for the reaction. Grill et al. identified kink defects along the step edges as the catalytically active sites for polymerization of $\alpha, \omega$ dibromoterfluorene molecules on $\mathrm{Au}(10,7,7) .{ }^{42}$

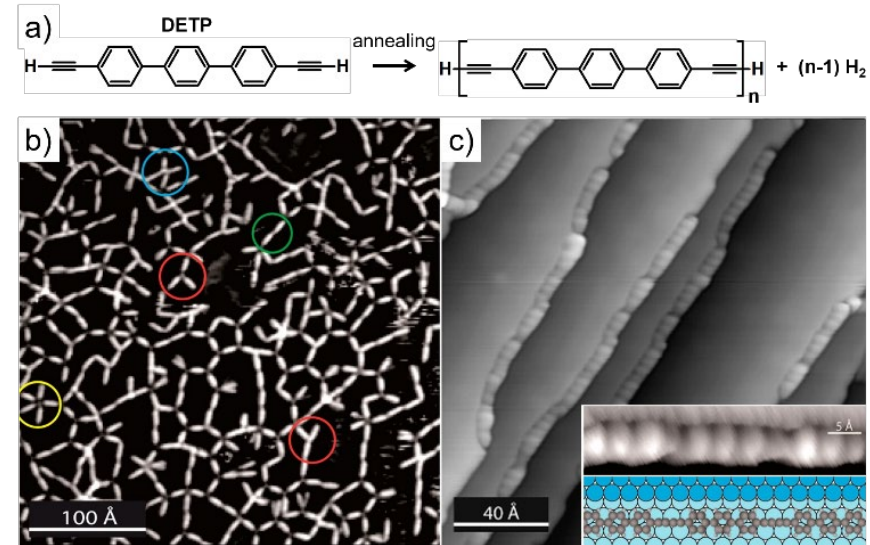

Fig. 16 (a) Alkyne homocoupling reaction between DETP precursors. (b) STM image showing a distribution of linear (green) and branched (red, blue, yellow) reaction products obtained after annealing DETP on $\mathrm{Ag}(111)$ at $400 \mathrm{~K}$ (c) STM image of extended 1D polymers organized along the substrate step edges of $\mathrm{Ag}(877)$ after annealing the sample to $450 \mathrm{~K}$. The inset displays a high-resolution image of part of a polymer along the lower substrate edge (top) along with a corresponding model (bottom). Reproduced from ref. 41 with permission from the American Chemical Society, copyright 2014.

Similar to stepped surfaces, the 1D constraint of missingrow reconstructions can be utilized to direct coupling reactions in a selective manner. As a result of molecular adsorption, and upon annealing, the anisotropic $\mathrm{Au}(110)$ surface is known to undergo a $(1 \times 3)$ reconstruction, characterized by $1.22 \mathrm{~nm}$ wide channels. In these narrow channels, diffusion of rod-like molecules is confined along the channel direction, and molecules can only interact with neighboring molecules in the same channel. As a result, coupling reactions selectively take place between the terminal sites of the molecules, yielding linear polymer chains instead of branched products. Using this strategy, long polyethylene chains or trans-configured polyphenyl wires could be obtained by surface-assisted dehydrogenative carbon-carbon coupling. ${ }^{43}, 44$ In addition to orienting the precursor molecules, adsorption within the channels may lower the barrier for $\mathrm{C}-\mathrm{H}$ activation compared to a planar (111) surface due to a higher degree of moleculesubstrate coordination. ${ }^{43}$

A particularly interesting template to direct on-surface reactions is a supergrating obtained by partial oxidation of the $\mathrm{Cu}(110)$ surface. By controlling the oxidation process, the template dimensions can be tuned providing access to various degrees of confinement. Using such tunable template, Fan et al. achieved a high degree of structural control over the Ullmann coupling reaction between 4,4"-dibromo-meta-terphenyl precursors ( $\mathrm{Br}_{2} \mathrm{MTP}$, Fig. 17). ${ }^{45} \mathrm{On} \mathrm{Cu}$ stripes exceeding $5.6 \mathrm{~nm}$, zigzag organometallic chains with a uniform length are predominantly formed. This structure is characterized by an alltrans configuration of the MTP moieties, similar to the polymeric chain motif obtained in the absence of any template. In contrast thereto, on narrower $\mathrm{Cu}$ stripes in the range of 2.6 to $5.6 \mathrm{~nm}$, organometallic macrocycles and trapezoid-wavelike chains are obtained exhibiting both cis and trans configured MTP moieties. In the ultimate case of $1.5 \mathrm{~nm}$ wide $\mathrm{Cu}$ stripes, 


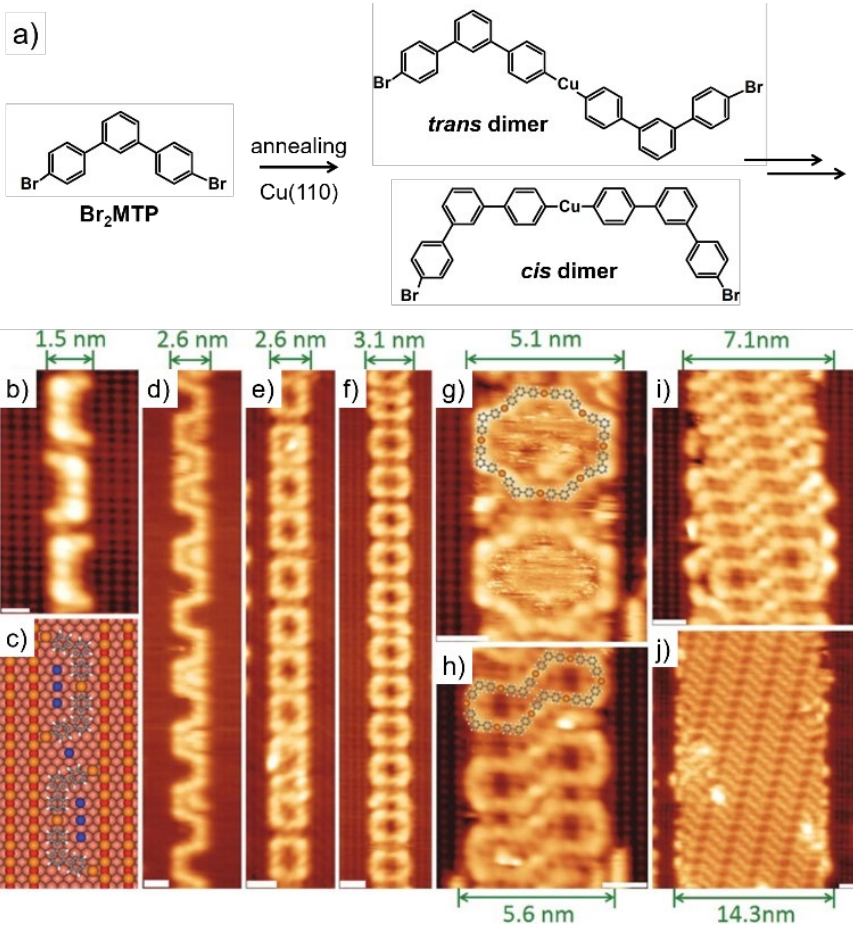

Fig. 17 Overview of the various products obtained from the Ullmann coupling of $\mathrm{Br}_{2} \mathrm{MTP}$ units on bare $\mathrm{Cu}$ stripes with different widths. (a) Molecular structure and Ullmann coupling reaction. (b) STM image of cis-configured dimers. (c) Molecular model; blue atoms represent single $\mathrm{Br}$ adatoms. (d) Trapezoid wavelike chains. $(e, f)$ Tetrameric macrocycles. $(g, h)$ Octameric macrocycles. (i,j) Zigzag organometallic chains with different widths. Reproduced from ref. 45 with permission from the American Chemical Society, copyright 2016

only cis-configured dimers can be formed. The transition from all-trans zigzag chains over macrocycles to cis-configured dimers is clearly induced by the spatial confinement effect imposed by the template dimensions. In addition, hydrogen bonding between the $\mathrm{Cu}-\mathrm{O}$ borders and hydrogen atoms of the MTP moieties are believed to stabilize the cis-configured motif on narrow stripes.

Besides the various pre-defined templates, one can also use linear polymer chains as a physical barrier for confining onsurface reactions. For instance, in the narrow grooves between in situ created poly(para-phenylene) chains on a $\mathrm{Au}(111)$ surface, graphene-like nanoribbons with embedded four- and eight-membered rings could successfully be obtained. ${ }^{46}$ Key to fabricate long nanoribbons is to avoid undesired side reactions between the ribbon precursor species by shielding them within the grooves.

\section{OD templates}

The topochemical polymerization of diacetylene derivatives on graphite has been the topic of many fundamental studies on onsurface reactivity. This reaction can be initiated by UV light or voltage pulses (for instance applied through the tip of an STM) to create a diradical state, after which the radicals propagate throughout the self-assembled network (Fig. 18a). The main advantage of using the STM tip rather than light to induce the reaction is the nanometer scale precision over the location of a)
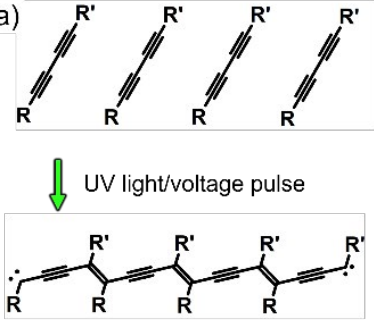

$\mathrm{R}=\left(\mathrm{CH}_{2}\right)_{8} \mathrm{COOH}$

$\mathrm{R}^{\prime}=\left(\mathrm{CH}_{2}\right)_{9} \mathrm{CH}_{3}(\mathrm{TCDA})$

$\mathrm{R}^{\prime}=\left(\mathrm{CH}_{2}\right)_{11} \mathrm{CH}_{3}(\mathrm{PCDA})$
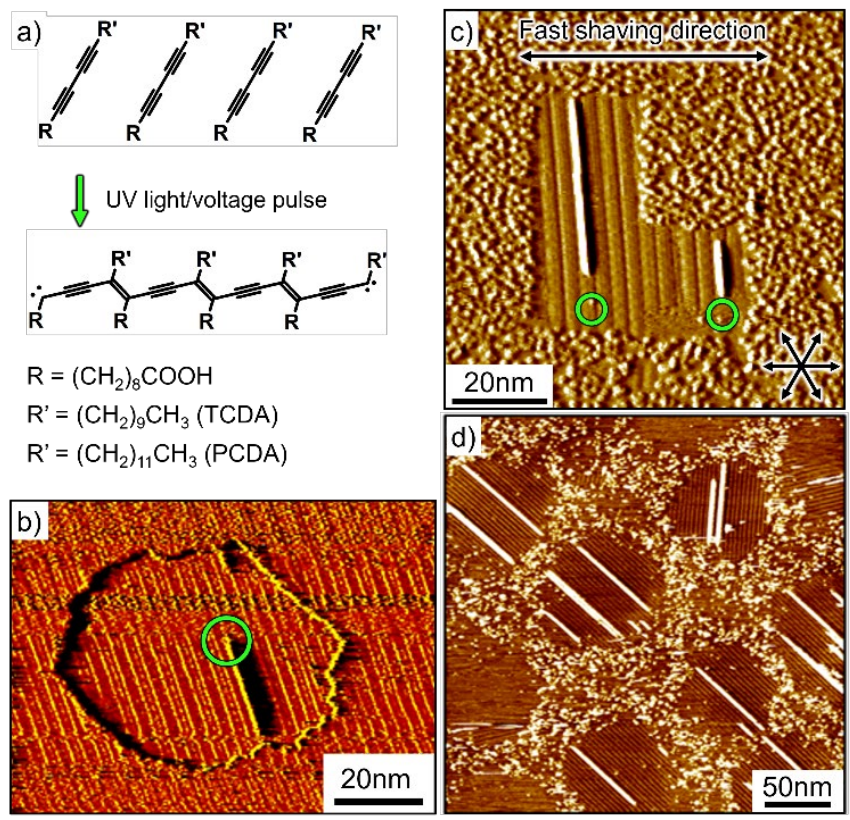

Fig. 18 (a) Diacetylene polymerization reaction. (b) STM image showing the confined polymerization of self-assembled 10,12-tricosadiynoic acid (TCDA) precursors in an etch pit corral on HOPG. The bright line corresponds to a polymer chain created by application of a voltage pulse at the location indicated by the green circle. Reproduced from ref. 47 with permission from the American Chemical Society, copyright 2005. (c) STM image of two polymer chains created by pulse-induced (green circles) polymerization of a self-assembled network of 10,12-pentacosadiynoic acid (PCDA) within a nanoshaved corral on covalently modified graphite. The fast shaving direction was oriented parallel to a graphite high-symmetry axis (black arrows). Reproduced from ref. 48 with permission from the Royal Society of Chemistry, copyright 2017. (d) STM image showing UV light induced polymerization of PCDA domains isolated within spontaneously formed corrals on HOPG. Reproduced from ref. 24 with permission from the American Chemical Society, copyright 2019.

chain initiation. Nonetheless, on bare graphite, the location of chain termination is determined by random quenching events. This lack of control over the degree of polymerization can, partially, be overcome by confining the diacetylene reactants to a surface template. Both etch pits and nanoshaved corrals were shown to be effective templates to restrict the reaction (Fig. $18 \mathrm{~b}, \mathrm{c}) .{ }^{47,} 48$ The latter approach also allowed to select the polymer orientation with respect to the substrate due to the alignment effect. On a larger scale, polydiacetylene synthesis could be controlled by light-induced polymerization inside nanocorrals obtained by electrografting from a mixture of diazonium salts (Fig. 18d). ${ }^{24}$

Next to controlling the product dimensions, confinement of reactants can also provide valuable insights into reaction mechanisms. For instance, the metal-catalyzed Ullmann coupling of aryl halides is generally agreed to proceed via a metal-organic intermediate, but the specific role of substrate atoms vs. adatoms in the formation of this intermediate is not clear. In order to clarify the mechanistic pathway, Judd et al. proposed to confine the reactant molecules, 1,3,5-tris(4iodophenyl)benzene (TIPB), to the pores of a self-assembled 


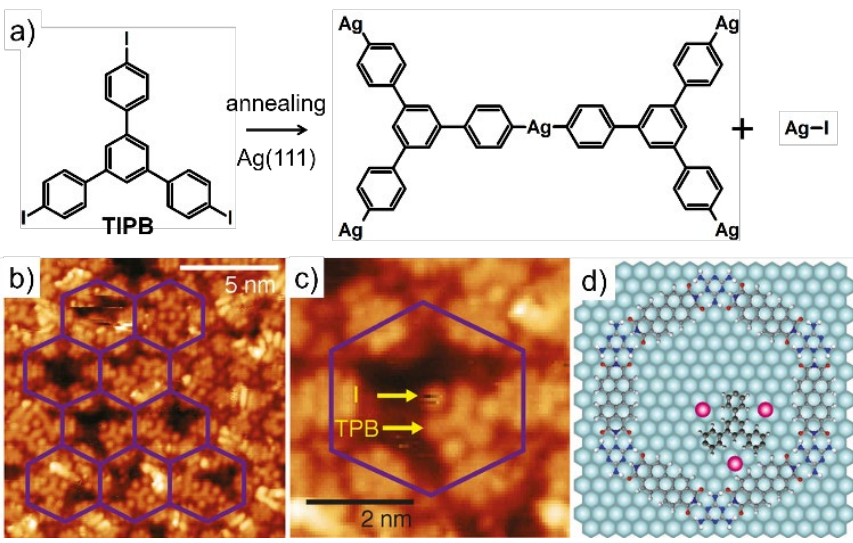

Fig. 19 (a) First step of the surface-assisted Ullmann coupling of TIPB yielding a metal-organic intermediate. $(b, c)$ Low temperature STM images showing the products obtained within a hexagonal porous network formed by 3,4,9,10-perylene tetracarboxylic acid diimide and melamine (highlighted in purple). Individual TPB fragments are surrounded by the cleaved iodine atoms. (d) Molecular model corresponding to (c). Reproduced from ref. 49 with permission from Wiley-VCH, copyright 2018.

molecular network on $\mathrm{Ag}(111)$ as shown in Fig. $19 .{ }^{49}$ By doing so, the TIPB molecules were denied access to freely diffusing adatoms, and any occurring reaction must be catalyzed directly by substrate atoms. Low-temperature STM imaging shows that the self-assembled pores can trap up to three reactant molecules, and that the carbon-iodine bonds have been catalytically cleaved. The iodine atoms are identified as the circular features arranged around the TPB species (Fig. 19c). However, even though the pore size is sufficiently large, no metal-organic intermediates are observed. This is in contrast to the non-templated synthesis, which is characterized by the formation of extended metal-organic frameworks. This discrepancy is attributed to the lack of Ag adatoms available to the reactants inside the pores, or to the presence of trapped iodine atoms that may alter the reactivity of the TPB species. In any case, it can be concluded that surface adatoms are not required for the initial step of the Ullmann coupling reaction, i.e. the carbon-iodine bond scission.

The pore dimensions in previous example are not much larger than the dimensions of the precursor molecules. Consequently, larger molecular templates are desirable to study the formation of more extended structures. Macromolecular porphyrin nanorings represent one of the largest molecular templates available to date, and are therefore interesting to be used for confined synthesis. Using a $13 \mathrm{~nm}$ wide nanoring comprising 40 porphyrin units on $\mathrm{Au}(111)$, patches of covalently bound TPB could be obtained by annealing the confined TIPB precursors to $110{ }^{\circ} \mathrm{C} .{ }^{50}$ However, the applicability of such templates is limited as the nanorings tend to break apart at the annealing temperature needed to induce the reaction.

\section{Conclusion}

This article provides an overview of the unique ways in which SAMN formation is influenced by lateral confinement in 2D. Nanoscale surface patterns limit the size and geometry to which assemblies can grow, and change their stability due to the often large number of molecules at the assembly periphery. As a result, a tendency for (I) optimal coverage of the available surface, and (II) stabilization by lateral interactions with the confining walls can be observed. This is most apparent on anisotropic surfaces, which often display preferred assembly orientations. In specific cases, polymorphism and 2D chirality can be influenced by the same factors. Significant effects have also been observed on the nucleation and growth behavior of 2D assemblies. In etch pit corrals for instance, the decreasing nucleation rate for smaller corral dimensions, and the lack of ordered structures in the most tight spaces suggests the existence of a critical nucleus size. In addition, the confining environment of nanoscale surface patterns provides an ideal platform to study and direct on-surface reactions and improve product selectivity. Nonetheless, while a variety of confinement effects have been described, the stability of the confined assemblies with respect to time, temperature, and concentration is often not addressed in detail. To get an indepth understanding of the mechanisms underlying confinement effects, future studies that systematically investigate these factors are highly desired.

Two main strategies for studying confinement were discussed, being patterned surfaces and SPL. While surface patterns are limited in terms of precise geometry, the patterns often propagate over macroscopic length scales. In the case of vicinal surfaces, the step-edge orientation is even retained over the entire sample surface. These characteristics of patterned surfaces provide clear advantages with regards to alignment of assemblies or reaction products, as well as for future scalability. Scanning probe lithography, on the other hand, can fabricate a variety of patterns on a single surface, allowing to directly assess geometry related aspects of confinement on a more fundamental level. For studying nucleation mechanisms, however, one must consider the limitations of each confinement strategy, and care should be taken when extrapolating the results to nucleation on open terraces. If significant interactions between the host structure and adsorbate molecules exist, the stability of emerging nuclei may be strongly impacted. Furthermore, impeded surface diffusion inside and to the confining environment can drastically impact the nucleation kinetics. In the case of in situ SPL, the possibility of tip-induced artefacts further complicate interpretation of the obtained results. In this regard, separating the shaving procedure from molecular self-assembly is expected to enhance the impact of SPL for studying 2D confinement.

Despite the above mentioned limitations, the insights gained from confinement studies can benefit the field of 2D supramolecular self-assembly in two ways. First, the ability to study nanoscopic assemblies can provide us with a wealth of information concerning the initial stages of 2D self-assembly. These studies may afford a better understanding of nucleation mechanisms, and ways to influence the nucleation and growth behavior via interactions with the host medium. Second, surface templates can make use of confinement effects to direct on-surface assemblies and reactions towards the desired morphology, which is of significant interest for engineering 
modified interfaces with improved (opto-)electronic properties and molecular recognition abilities. This may open new possibilities for applications in organic electronics, photovoltaics, catalysis, or as templates for heterogeneous crystallization.

\section{Conflicts of interest}

There are no conflicts to declare.

\section{Acknowledgements}

The authors gratefully acknowledge financial support from the Fund of Scientific Research Flanders (FWO), the KU Leuven Internal Funds. L.V. thanks the FWO for the fellowship received.

\section{Notes and references}

1. G. T. Rengarajan, D. Enke, M. Steinhart and M. Beiner, Phys. Chem. Chem. Phys., 2011, 13, 21367-21374.

2. Y. Diao, T. Harada, A. S. Myerson, T. A. Hatton and B. L. Trout, Nat. Mater., 2011, 10, 867-871.

3. B. D. Hamilton, I. Weissbuch, M. Lahav, M. A. Hillmyer and M. D. Ward, J. Am. Chem. Soc., 2009, 131, 2588-2596.

4. B. D. Hamilton, J. M. Ha, M. A. Hillmyer and M. D. Ward, Acc. Chem. Res., 2012, 45, 414-423.

5. Q. Jiang and M. D. Ward, Chem. Soc. Rev., 2014, 43, 20662079.

6. F. C. Meldrum and C. O'Shaughnessy, Adv. Mater., 2020, 32.

7. R. Otero, Y. Naitoh, F. Rosei, P. Jiang, P. Thostrup, A Gourdon, E. Laegsgaard, I. Stensgaard, C. Joachim and F. Besenbacher, Angew. Chem., 2004, 43, 2092-2095.

8. D. L. Patrick, V. J. Cee and T. P. Beebe, Science, 1994, 265 231-234.

9. J. Greenwood, T. H. Phan, Y. Fujita, Z. Li, O. Ivasenko, W. Vanderlinden, H. Van Gorp, W. Frederickx, G. Lu, K. Tahara, Y. Tobe, H. Uji-i, S. F. L. Mertens and S. De Feyter, ACS Nano, 2015, 9, 5520-5535.

10. M. Sleutel, J. Lutsko, A. E. S. Van Driessche, M. A. DuranOlivencia and D. Maes, Nat. Commun., 2014, 5.

11. J. J. Chen, E. B. Zhu, J. Liu, S. Zhang, Z. Y. Lin, X. F. Duan, H. Heinz, Y. Huang and J. J. De Yoreo, Science, 2018, 362, 1135-1139.

12. I. Chizhov, G. Scoles and A. Kahn, Langmuir, 2000, 16, 4358-4361.

13. D. G. de Oteyza, E. Barrena, H. Dosch and Y. Wakayama, Phys. Chem. Chem. Phys., 2009, 11, 8741-8744.

14. J. Wang, I. Kaur, B. Diaconescu, J. M. Tang, G. P. Miller and K. Pohl, ACS Nano, 2011, 5, 1792-1797.

15. M. E. Canas-Ventura, W. Xiao, D. Wasserfallen, K. Mullen, H. Brune, J. V. Barth and R. Fasel, Angew. Chem., 2007, 46, 1814-1818.

16. J. Kroger, N. Neel, H. Jensen, R. Berndt, R. Rurali and N. Lorente, J. Phys.: Condens. Matter, 2006, 18.

17. F. Cicoira, J. A. Miwa, D. F. Perepichka and F. Rosei, J. Phys. Chem. A, 2007, 111, 12674-12678.

18. H. P. Chang and A. J. Bard, J. Am. Chem. Soc., 1990, 112, 4598-4599.
19. D. L. Patrick, V. J. Cee and T. P. Beebe, J. Phys. Chem., 1996, 100, 8478-8481.

20. D. E. Hooks, C. M. Yip and M. D. Ward, J. Phys. Chem. B, 1998, 102, 9958-9965.

21. A. Tracz, A. Stabel and J. P. Rabe, Langmuir, 2002, 18 9319-9326.

22. D. L. Patrick, V. J. Cee, T. J. Purcell and T. P. Beebe, Langmuir, 1996, 12, 1830-1835.

23. H. Van Gorp, P. Walke, A. M. Braganca, J. Greenwood, O. Ivasenko, B. E. Hirsch and S. De Feyter, ACS Appl. Mater. Interfaces, 2018, 10, 12005-12012.

$24 . \quad$ T. H. Phan, H. Van Gorp, Z. Li, T. M. T. Huynh, Y. Fujita, L. Verstraete, S. Eyley, W. Thielemans, H. Uji-i, B. E. Hirsch, S. F. L. Mertens, J. Greenwood, O. Ivasenko and S. De Feyter, ACS Nano, 2019, 13, 5559-5571.

25. A. M. Braganca, J. Greenwood, O. Ivasenko, H. Phan, K. Mullen and S. De Feyter, Chem. Sci., 2016, 7, 7028-7033.

26. A. M. Braganca, B. E. Hirsch, A. Sanz-Matias, Y. Hu, P. Walke, K. Tahara, J. N. Harvey, Y. Tobe and S. De Feyter, J. Phys. Chem. C, 2018, 122, 24046-24054.

27. S. Xu and G. Y. Liu, Langmuir, 1997, 13, 127-129.

28. M. Liu, N. A. Amro and G. Y. Liu, Annu. Rev. Phys. Chem., 2008, 59, 367-386.

29. S. Xu, P. E. Laibinis and G. Y. Liu, J. Am. Chem. Soc., 1998, 120, 9356-9361.

$30 . \quad J$. J. Yu, Y. H. Tan, X. Li, P. K. Kuo and G. Y. Liu, J. Am. Chem. Soc., 2006, 128, 11574-11581.

31. S. Ryu and G. C. Schatz, J. Am. Chem. Soc., 2006, 128 11563-11573.

32. D. Takajo, T. Nemoto, H. Kurata, S. Isoda, H. Ozaki and Y. Mazaki, Thin Solid Films, 2003, 438, 428-432.

33. D. Takajo, T. Nemoto, O. Franco, H. Kurata and S. Isoda, Jpn. J. Appl. Phys., 2006, 45, 2091-2094.

34. L. Verstraete, J. Greenwood, B. E. Hirsch and S. De Feyter, ACS Nano, 2016, 10, 10706-10715.

35. L. Verstraete, J. Smart, B. E. Hirsch and S. De Feyter, Phys. Chem. Chem. Phys., 2018, 20, 27482-27489.

36. F. Stevens, D. Buehner and T. P. Beebe, J. Phys. Chem. B, 1997, 101, 6491-6496.

37. J. Seibel, L. Verstraete, B. E. Hirsch, A. M. Bragança and S. De Feyter, J. Am. Chem. Soc., 2018, 140, 11565-11568.

38. J. Seibel, D. B. Amabilino and S. De Feyter, Angew. Chem., 2019, 58, 12964-12968.

39. Y. Hu, A. M. Braganca, L. Verstraete, O. Ivasenko, B. E. Hirsch, K. Tahara, Y. Tobe and S. De Feyter, Chem. Commun., 2019, 55, 2226-2229.

40. L. Verstraete, P. Szabelski, A. M. Bragança, B. E. Hirsch and S. De Feyter, Chem. Mater., 2019, 31, 6779-6786.

41. B. Cirera, Y. Q. Zhang, J. Bjork, S. Klyatskaya, Z. Chen, M. Ruben, J. V. Barth and F. Klappenberger, Nano Lett., 2014, 14, 1891-1897.

42. A. Saywell, J. Schwarz, S. Hecht and L. Grill, Angew. Chem. 2012, 51, 5096-5100.

43. D. Y. Zhong, J. H. Franke, S. K. Podiyanachari, T. Blomker, H. M. Zhang, G. Kehr, G. Erker, H. Fuchs and L. F. Chi, Science, 2011, 334, 213-216.

44. Z. Y. Cai, L. M. She, L. Q. Wu and D. Y. Zhong, J. Phys. Chem. C, 2016, 120, 6619-6624.

45. Q. T. Fan, J. Y. Dai, T. Wang, J. Kuttner, G. Hilt, J. M. Gottfried and J. F. Zhu, ACS Nano, 2016, 10, 3747-3754. 
46. M. Z. Liu, M. X. Liu, L. M. She, Z. Q. Zha, J. L. Pan, S. C. Li, T.

Li, Y. Y. He, Z. Y. Cai, J. B. Wang, Y. Zheng, X. H. Qiu and D.

Y. Zhong, Nat. Commun., 2017, 8.

47. S. P. Sullivan, A. Schmeders, S. K. Mbugua and T. P. Beebe, Langmuir, 2005, 21, 1322-1327.

48. L. Verstraete, B. E. Hirsch, J. Greenwood and S. De Feyter, Chem. Commun., 2017, 53, 4207-4210.

49. C. J. Judd, N. R. Champness and A. Saywell, Chem. -Eur. J., 2018, 24, 56-61.

50. C. J. Judd, D. Kondratuk, H. L. Anderson and A. Saywell, Sci. Rep., 2019, 9. 